\title{
Gallbladder Cancer: We Need to Do Better!
}

\author{
Parul J. Shukla, MBBS, MS, FRCS ${ }^{1}$ and Savio G. Barreto, MBBS, MS ${ }^{2}$ \\ ${ }^{1}$ Gastrointestinal Surgical Oncology, Tata Memorial Hospital, Mumbai, India; ${ }^{2}$ General and Digestive Surgery, \\ Flinders Medical Centre, Adelaide, SA, Australia
}

Gallbladder cancer is associated with a dismal prognosis, with a 5-year survival rate of $<5 \%$ for those patients with disease not amenable to surgery. ${ }^{1,2}$ In some parts of the world, the incidence is comparatively high and contributes to a social disaster as a result of its associated poor outcome. Even among patients who undergo radical surgical resection, the reported median survival across all stages is $35 \%$ to $38 \%$. $^{2,3}$

The surgical management of gallbladder cancer has always been controversial. Surgeons across the world have repeatedly stressed the need for a complete surgical resection and have demonstrated improved survival in patients who underwent radical resection. ${ }^{2-6}$ Guidelines for the surgical management of gallbladder cancer have been formulated on the basis of the available evidence. ${ }^{7-9}$ Despite this, it now appears that one of the biggest problems in gallbladder cancer is the lack of performance of the correct surgery in the first place. ${ }^{10-15}$

An offshoot of the problem of inadequate surgery for gallbladder cancer is the misuse of the term incidental gallbladder cancer and the resulting mismanagement of the patients included in this group. The term incidental was coined to denote a histological surprise of gallbladder cancer in a patient treated with simple cholecystectomy for cholecystitis or gallbladder polyps. However, we conducted a study and found that up to $50 \%$ of patients with disease labeled as incidental gallbladder cancers in the particular series we were assessing actually had a radiological diagnosis suspicious for gallbladder cancer, despite which the patients were treated solely with a simple cholecystectomy. ${ }^{10}$

Another problem with the surgical management of gallbladder cancer has been the interchangeable use of the

(C) Society of Surgical Oncology 2009

Published Online: 9 June 2009

P. J. Shukla, MBBS, MS, FRCS

e-mail: pjshukla@doctors.org.uk terms extended, radical, and aggressive surgery when describing the optimal surgery for gallbladder cancer. Like any other solid organ cancer, the aim of surgery in gallbladder cancer should be to achieve complete tumor clearance with a stepwise escalation of the extent of radicality determined by on-table surgical acumen coupled with information gathered preoperatively via imaging. ${ }^{16}$

Considering that surgery remains the treatment modality with the best reported outcomes, the worries with regard to the management of gallbladder cancer are as follows: Is the algorithm for the management of this cancer unclear? Does the treatment algorithm need to be more explicit? What can we do to improve outcomes in gallbladder cancer?

Although the relative incidence of gallbladder cancer differs widely across countries, the algorithm needs to be understood by all surgeons treating diseases of the gallbladder. In patients with a preoperative diagnosis of gallbladder cancer, it is imperative that the patient be treated with a cholecystectomy with en-bloc hepatic resection with lymphadenectomy with or without bile duct resection. The extent of the hepatic resection has been reported to vary from a wedge of the gallbladder bed to major right lobe of liver resections. Although the debate on the extent of liver resection continues, the basis for including the gallbladder as part of the en-bloc resection of the gallbladder is to prevent desecration of the subserosal plane of the gallbladder (as is done in a simple cholecystectomy), which could aid tumor cell spread, and also to include potential micrometastases via the lymphatics of the gallbladder traversing the gallbladder bed. ${ }^{17}$ The rationale for including a bile duct resection should be based on the cause of the gallbladder cancer, with routine excision of the bile duct performed for patients with anomalous pancreatic bile duct junctions. In all other patients, the excision of the bile duct should be performed only when involved or when surgically indicated. Such indications include direct bile duct involvement by the cancer, and positive cystic duct 
margin in which a revision of the margin would entail encroaching on the bile duct. In addition, the procedure might be performed to aid lymphadenectomy, especially in patients undergoing repeat surgery (for incidental gallbladder cancer) in which the lymphadenectomy results in devascularization of the bile duct.

D'Angelica et al., in their recently published analysis on the effect of the extent of resection on disease-specific survival and perioperative outcomes, demonstrated poor outcomes after overaggressive surgeries such as major hepatectomies and bile duct resections performed when not clinically indicated. ${ }^{18}$ This reaffirms the basic oncologic tenet that cancer survival is determined by tumor biology and not extent of resection. ${ }^{19}$

If the results of radiologic investigations are suspicious for or suggest a potential gallbladder cancer, the patient should undergo a cholecystectomy with intraoperative frozen section..$^{10}$ If the diagnosis of malignancy is confirmed, in tumors that exceed the $\mathrm{T} 1 \mathrm{~b}$ classification, the procedure should be completed with a hepatic resection with hepatoduodenal lymphadenectomy with or without bile duct resection. ${ }^{6,9}$ There is no justification for performing a simple cholecystectomy in patients with gallbladder cancer who have tumors classified as greater than $\mathrm{T} 1 \mathrm{~b}$ - and this message needs to be clear. In T1a tumors, a simple cholecystectomy appears to constitute an adequate resection.

In incidental or missed gallbladder cancers, patients who have undergone only a simple cholecystectomy and who have tumors that are stage T1b or more should be considered for radical repeat resection after a thorough workup to exclude any evidence of metastasis. ${ }^{6}$ The use of positron emission tomography imaging as a complementary tool to computed tomography of the abdomen has been demonstrated, especially its use in ruling out metastasis in the body outside the abdominal cavity. ${ }^{20}$ Radical repeat resection in these patients has been shown to be associated with better survival compared with those who only undergo a simple cholecystectomy. ${ }^{4}$

Although long-term outcomes after radical repeat resection for true incidental gallbladder cancers are reported to be similar as those of primary radical surgery, there are no data comparing outcomes of radical repeat resection in patients whose tumors were truly incidental compared with patients whose tumors were classified as missed, potential, or suspicious.

The message is clear: if we want to improve outcomes in gallbladder cancer, we need to get it right the first time.

\section{REFERENCES}

1. Shukla PJ, Neve R, Barreto SG, et al. A new scoring system for gallbladder cancer (aiding treatment algorithm): an analysis of 335 patients. Ann Surg Oncol. 2008;15:3132-7.
2. Fong Y, Jarnagin W, Blumgart LH. Gallbladder cancer: comparison of patients presenting initially for definitive operation with those presenting after prior noncurative intervention. Ann Surg. 2000;232:557-69.

3. Dixon E, Vollmer C, Sahajpal A, et al. An aggressive surgical approach leads to improved survival in patients with gallbladder cancer. Ann Surg. 2005;241:385-94.

4. Foster JM, Hoshi H, Gibbs JF, et al. Gallbladder cancer: defining the indications for primary radical resection and radical reresection. Ann Surg Oncol. 2007;14:833-40.

5. Chan SY, Poon RT, Lo CM, et al. Management of carcinoma of the gallbladder: a single-institution experience in 16 years. J Surg Oncol. 2008;97:156-64.

6. Shukla PJ, Barreto G, Kakade A, Shrikhande SV. Revision surgery for gallbladder cancer: factors influencing operability and further evidence for T1b tumours. HPB (Oxford). 2008;10:43-7.

7. Kondo S, Takada T, Miyazaki M; Japanese Association of Biliary Surgery; Japanese Society of Hepato-Biliary-Pancreatic Surgery; Japan Society of Clinical Oncology. Guidelines for the management of biliary tract and ampullary carcinomas: surgical treatment. J Hepatobiliary Pancreat Surg. 2008;15:41-4.

8. Shukla PJ, Barreto SG. Approach to carcinoma of the gallbladder. In: Roshan Lall Gupta, editor. Recent Advances in Surgery 11. Indian ed. New Delhi: Jaypee Brothers; 2009. p. 188-207.

9. NCCN Guidelines for Hepatobiliary Cancers. Available at: http:// www.nccn.org/professionals/physician_gls/PDF/hepatobiliary. pdf. Accessed 5 June 2009.

10. Shukla PJ, Barreto G, Neve R, et al. Can we do better than incidental gallbladder cancer? Hepatogastroenterology. 2007;54: 2184-5.

11. Kiran RP, Pokala N, Dudrick SJ. Incidence pattern and survival for gallbladder cancer over three decades-an analysis of 10301 patients. Ann Surg Oncol. 2007;14:827-32.

12. Kayahara M, Nagakawa T. Recent trends of gallbladder cancer in Japan: an analysis of 4770 patients. Cancer. 2007;110:572-80.

13. Coburn NG, Cleary SP, Tan JC, Law CH. Surgery for gallbladder cancer: a population based-analysis. J Am Coll Surg. 2008;207: 371-82.

14. Jensen EH, Abraham A, Habermann EB, et al. A critical analysis of the surgical management of early-stage gallbladder cancer in the United States. J Gastrointest Surg. 2009;13:722-7.

15. Wright BE, Lee CC, Iddings DM, et al. Management of T2 gallbladder cancer: are practice patterns consistent with national recommendations? Am J Surg 2007;194:820-5.

16. Pawlik TM, Gleisner AL, Vigano L, et al. Incidence of finding residual disease for incidental gallbladder carcinoma: implications for re-resection. J Gastrointest Surg. 2007;11:1478-86.

17. Mochinaga M. Carcinoma of the gallbladder: a study of its modes of spread and surgical treatment. Nippon Geka Gakkai Zasshi 1983;16:1334-44.

18. D'Angelica M, Dalal KM, DeMatteo RP, et al. Analysis of the extent of resection for adenocarcinoma of the gallbladder. Ann Surg Oncol. 2009;16:806-16.

19. Pawlik TM, Choti MA. Biology dictates prognosis following resection of gallbladder carcinoma: sometimes lees is more. Ann Surg Oncol. 2009;16:787-8.

20. Shukla PJ, Barreto SG, Arya S, et al. Does PET-CT scan have a role prior to radical re-resection for incidental gallbladder cancer. HPB (Oxford) . 2008;10:439-45. 